\title{
addhaz: Contribution of Chronic Diseases to the Disability Burden Using R
}

by Renata Tiene de Carvalho Yokota, Caspar WN Looman, Wilma Johanna Nusselder, Herman Van Oyen and Geert Molenberghs

\begin{abstract}
The increase in life expectancy followed by the burden of chronic diseases contributes to disability at older ages. The estimation of how much chronic conditions contribute to disability can be useful to develop public health strategies to reduce the burden. This paper introduces the R package addhaz, which is based on the attribution method (Nusselder and Looman, 2004) to partition disability into the additive contributions of diseases using cross-sectional data. The R package includes tools to fit the additive hazard model, the core of the attribution method, to binary and multinomial outcomes. The models are fitted by maximizing the binomial and multinomial log-likelihood functions using constrained optimization. Wald and bootstrap confidence intervals can be obtained for the parameter estimates. Also, the contribution of diseases to the disability prevalence and their bootstrap confidence intervals can be estimated. An additional feature is the possibility to use parallel computing to obtain the bootstrap confidence intervals. In this manuscript, we illustrate the use of addhaz with several examples for the binomial and multinomial models, using the data from the Brazilian National Health Survey, 2013.
\end{abstract}

\section{Introduction}

The increase in longevity observed worldwide is usually followed by the burden of chronic diseases, which are among the leading causes of disability late in life (Beard et al., 2016). Disability has become a public health priority due to its adverse effects on health outcomes and quality of life, resulting in increased costs of health care (Yang et al., 2014). Therefore, the identification of which chronic diseases are the main contributors to the disability burden plays an important role in the formulation of public health response to population aging (Klijs et al., 2011).

Although prospective studies are better suited to establish the causal relationship between chronic diseases and disability, they are costly and usually with limited sample size. Alternatively, crosssectional data has been widely used to investigate the association of chronic diseases and disability. Among the methods based on cross-sectional data, the attribution method proposed by Nusselder and Looman (2004) has the advantage of partitioning the disability prevalence into the additive contributions of chronic diseases, taking into account multimorbidity and that disability can be present even in the absence of chronic diseases. The method was originally proposed for binary outcomes, but it was recently extended to multicategory response variables (Yokota et al., 2017) and it is based on the binomial and multinomial additive hazard models, respectively. The use of non-canonical link functions in the models imposes a constraint on the linear predictor, which limits the use of standard statistical software to fit the models, such as glm in R or proc GLM in SAS (SAS Institute Inc., 2008). Despite this practical difficulty, the attribution method for binary outcomes has been widely used previously with data from the Netherlands (Nusselder and Looman, 2004; Klijs et al., 2011), Belgium (Nusselder et al., 2005; Yokota et al., 2015b), Germany (Strobl et al., 2013), China (Chen et al., 2013), and Brazil (Yokota et al., 2016), owing to the development of the software in R to fit the binomial model and to estimate the contribution of diseases to the disability prevalence by Nusselder and Looman (2010) for non-R users, which is available upon request to the authors (w.nusselder@erasmusmc.nl).

In this manuscript we present the $R$ package addhaz, which is an extension of the $R$ software developed by Nusselder and Looman (2010), offering an open-source implementation of the binomial and multinomial additive hazard models. The R functions can also be used to calculate the contribution of chronic diseases to the disability burden for both models.

This paper is structured as follows. In Section 2, a brief description of the attribution method is presented, followed by the definition of the binomial and multinomial additive hazard models. Section 3 introduces some features and options of addhaz. The existing alternative software to fit the binomial and multinomial models is discussed in Section 4 . Examples of how to use the $\mathrm{R}$ functions to fit the models and to estimate contributions are shown in Section 5, using the data of the 2013 Brazilian National Health Survey (BNHS). The main advantages and disadvantages of the attribution method and addhaz are discussed in Section 6. Finally, conclusions and recommendations for future research are outlined in Section 7. 


\section{Attribution method}

Analogous to the mortality analysis, in which a single disease is assigned as underlying cause of death in the death certificate, the attribution method aims to assess the probability that a single reported disease was the cause of disability in a survey, taking into account that individuals can report more than one disease (multimorbidity) and that disability can be present without any reported diseases (Nusselder and Looman, 2004, 2010).

In the attribution method, the disability that is not associated with any disease included in the analysis is attributed to "background". The background can represent the effect of age-related losses in functioning; underreporting and underdiagnosed diseases; and other causes of disability that were not included in the survey or analysis. More details about the attribution method can be found elsewhere (Nusselder and Looman, 2004, 2010).

The following assumptions are required to fit the binomial and multinomial additive hazard models to cross-sectional data: (i) disability is caused by the diseases that are still present in the time of the survey and the background; (ii) the estimated cross-sectional cumulative rates reflect the transition rates that would have been estimated with longitudinal data (stationarity assumption); (iii) the recovery rate is zero; (iv) the ratio of the cause-specific cumulative rates to the overall cumulative rate is constant over time (proportionality assumption); (v) the start of the time (age) at risk to become disabled is the same for all diseases; (vi) individuals from the same age group are exposed to the same cumulative rate of disability for background; (vii) diseases and background act as independent competing causes of disability (Nusselder and Looman, 2004, 2010).

\section{Binomial additive hazard model}

For binary outcomes, the binomial additive hazard model is defined as:

$$
\begin{aligned}
& y_{i} \sim \operatorname{Bernoulli}\left(\pi_{i}\right) \\
& \pi_{i}=1-\exp \left(-\eta_{i}\right) \\
& \eta_{i}=\alpha_{a_{i}}+\sum_{d=1}^{m} \beta_{d} X_{d i}
\end{aligned}
$$

where $y_{i}$ is the binary disability outcome; $\pi_{i}$ is the disability probability for individual $i ; \eta_{i}$ is the linear predictor representing the overall cumulative rate (or cumulative hazard) of disability for individual $i$; $a_{i}$ denotes the age group of individual $i$ (with $f$ age groups, $a_{i}$ can only get values between $1, \ldots, f$ ); $\alpha_{a}$ is the cumulative rate of disability for background by age group $a(a=1, \ldots, f) ; \beta_{d}$ is the cumulative rate of disability (or disabling impact) for disease $d(1, \ldots, m)$; and $X_{d i}$ is the indicator variable for disease $d$ and individual $i$.

A linear inequality constraint is applied to the linear predictor $\left(\eta_{i} \geq 0\right)$ to ensure that $\pi_{i}$ lies between $(0,1)$. The standard errors $(S E)$ for the regression coefficients are estimated based on the inverse of the observed information matrix. The 95\% Wald confidence intervals (Wald CI) can be obtained using the standard errors described above (Wald CI) as showed in 2 or via bootstrapping (Efron and Tibshirani, 1994).

$$
\begin{aligned}
& 95 \% \text { Wald CI }=\widehat{\alpha}_{a} \pm 1.96(\widehat{S E}) \\
& 95 \% \text { Wald CI }=\widehat{\beta}_{d} \pm 1.96(\widehat{S E})
\end{aligned}
$$

\section{Multinomial additive hazard model}

The multinomial additive hazard model is an extension of the binomial model:

$$
\begin{aligned}
y_{i j} & \sim \operatorname{Multinomial}\left(1, \Pi_{i}\right) \\
\pi_{i j} & =\left[1-\exp \left(-\sum_{q=1}^{c} \eta_{i q}\right)\right]\left(\frac{\eta_{i j}}{\sum_{q=1}^{c} \eta_{i q}}\right) \\
\eta_{i j} & =\alpha_{a_{i} j}+\sum_{d=1}^{m} \beta_{d j} X_{d i}
\end{aligned}
$$

where $y_{i j}$ is the multinomial response variable (disability) with one independent observation and vector of disability probabilities $\Pi_{i}=\left(\pi_{i 0}, \ldots, \pi_{i j}, \ldots, \pi_{i c}\right)$ per individual $i ; \pi_{i j}$ is the probability of disability category $j$ for individual $i ; \eta_{i j}$ is the linear predictor (overall cumulative rate) for disability category $j$ and individual $i$; $a_{i}$ denotes the age group of individual $i$ (with $f$ age groups, $a_{i}$ can only 
get values between $1, \ldots, f) ; \alpha_{a j}$ is the cumulative rate of disability category $j$ for background by age group $a(a=1, \ldots, f) ; \beta_{d j}$ is the cumulative rate of disability category $j$ or disabling impact for disease $d(1, \ldots, m)$; and $X_{d i}$ is the indicator variable for disease $d$ and individual $i$.

Besides the inequality constraint in the linear predictor $\eta_{i j} \geq 0$, an additional constraint is required: $\sum_{j=1}^{c} \pi_{i j}<1$, to ensure that all $\pi_{i j}>0$. Similar to the binomial case, the standard errors are estimated by the inverse of the observed information matrix and the $95 \%$ Wald CI and bootstrap percentile confidence intervals (bootstrap CI) can be obtained using addhaz.

\section{Contribution of chronic diseases and background to the disability prevalence}

The attribution of disability to chronic diseases depends on the disease prevalence $\left(X_{d}\right)$ and the disabling impacts of the diseases $\left(\beta_{d}\right.$ and $\beta_{d j}$ ) (Nusselder and Looman, 2004, 2010). The contribution of chronic diseases and background to the disability prevalence can be calculated in five steps for both binary and multicategory response variables.

\section{Binary case}

For the binary case, the cause-specific disability probabilities for individual $i$ and each chronic condition $\left(\widehat{D}_{d i}\right)$ and the background $\left(\widehat{B}_{i}\right)$ are estimated based on the proportionality assumption, analogous to the competing risks setting in mortality analysis (Manton and Stallard, 1984; Chiang, 1991):

$$
\begin{aligned}
& \widehat{D}_{d i}=\widehat{\pi}_{i}\left(\frac{\widehat{\beta}_{d} X_{d i}}{\widehat{\eta}_{i}}\right) \\
& \widehat{B}_{i}=\widehat{\pi}_{i}\left(\frac{\widehat{\alpha}_{a i}}{\widehat{\eta}_{i}}\right)
\end{aligned}
$$

Next, the number of disabled individuals by each disease $\left(\widehat{N}_{d}\right)$ and background $\left(\widehat{N}_{b}\right)$ are estimated as:

$$
\begin{aligned}
& \widehat{N}_{d}=\sum_{i=1}^{n} \widehat{D}_{d i} \\
& \widehat{N}_{b}=\sum_{i=1}^{n} \widehat{B}_{i}
\end{aligned}
$$

The absolute contribution of each disease $\left(\widehat{A C}_{d}\right)$ and background $\left(\widehat{A C}_{b}\right)$ to the total disability prevalence is obtained by:

$$
\begin{aligned}
& \widehat{A C}_{d}=\frac{\widehat{N}_{d}}{n} \\
& \widehat{A C}_{b}=\frac{\widehat{N}_{b}}{n}
\end{aligned}
$$

The absolute contribution of background and diseases defined above sum to the disability prevalence $(\widehat{P})$ :

$$
\widehat{P}=\widehat{A C}_{b}+\sum_{d=1}^{m} \widehat{A C}_{d}
$$

Finally, the relative contribution of diseases $\left(\widehat{R C}_{d}\right)$ and background $\left(\widehat{R C}_{b}\right)$ to the disability prevalence is estimated by:

$$
\begin{aligned}
& \widehat{R C}_{d}=\frac{\widehat{A C}_{d}}{\widehat{P}} \\
& \widehat{R C}_{b}=\frac{\widehat{A C}_{b}}{\widehat{P}}
\end{aligned}
$$

The relative contributions $\left(\widehat{R C}_{d}\right.$ and $\left.\widehat{R C}_{b}\right)$ sum to 1 .

\section{Multinomial case}

Analogous to the binomial case, the contribution of chronic diseases to the disability prevalence for multinomial outcomes can be obtained in five steps for each category $j$ of the outcome: 
$i$ :

1. Cause-specific disability probabilities for each disease $\left(\widehat{D}_{d i j}\right)$ and background $\left(\widehat{B}_{i j}\right)$ for individual

$$
\begin{aligned}
& \widehat{D}_{d i j}=\widehat{\pi}_{i j}\left(\frac{\widehat{\beta}_{d j} X_{d i}}{\widehat{\eta}_{i j}}\right) \\
& \widehat{B}_{i j}=\widehat{\pi}_{i j}\left(\frac{\widehat{\alpha}_{a_{i j}}}{\widehat{\eta}_{i j}}\right)
\end{aligned}
$$

2. Number of disabled individuals by each disease $\left(\widehat{N}_{d j}\right)$ and background $\left(\widehat{N}_{b j}\right)$ :

$$
\begin{aligned}
& \widehat{N}_{d j}=\sum_{i=1}^{n} \widehat{D}_{d i j} \\
& \widehat{N}_{b j}=\sum_{i=1}^{n} \widehat{B}_{i j}
\end{aligned}
$$

3. Absolute contribution of each disease $\left(\widehat{A C}_{d j}\right)$ and background $\left(\widehat{A C}_{b j}\right)$ to the total disability prevalence:

$$
\begin{aligned}
& \widehat{A C}_{d j}=\frac{\widehat{N}_{d j}}{n} \\
& \widehat{A C}_{b j}=\frac{\widehat{N}_{b j}}{n}
\end{aligned}
$$

4. Total disability prevalence $\left(\widehat{P}_{j}\right)$ :

$$
\widehat{P}_{j}=\widehat{A C}_{b j}+\sum_{d=1}^{m} \widehat{A C}_{d j}
$$

5. Relative contribution of diseases $\left(\widehat{R C}_{d j}\right)$ and background $\left(\widehat{R C}_{b j}\right)$ to the disability prevalence:

$$
\begin{aligned}
& \widehat{R C}_{d j}=\frac{\widehat{A C}_{d j}}{\widehat{P}_{j}} \\
& \widehat{R C}_{b j}=\frac{\widehat{A C}_{b j}}{\widehat{P}_{j}}
\end{aligned}
$$

The absolute contributions defined in equations 11 sum to the prevalence of disability for each category $j$ and the relative contributions defined in equations 13 sum to 1 for each disability category $j$. The confidence intervals for the absolute and relative contributions for the binary and multinomial cases can be estimated via bootstrapping (Efron and Tibshirani, 1994) in addhaz.

\section{Features of addhaz}

In this section, a brief explanation about the constrained optimization, the parallel option to obtain the bootstrap CI for the parameter estimates, and the option to perform the likelihood ratio test for model selection is provided.

\section{Constrained optimization}

The binomial and multinomial additive hazard models are generalized linear models with noncanonical link functions $\eta_{i}=\log \left(\frac{1}{1-\pi_{i}}\right)$ for the binomial model and $\eta_{i j}=\left[-\log \left(1-\sum_{q=1}^{c} \pi_{i q}\right)\right]\left(\frac{\pi_{i j}}{\sum_{q=1}^{c} \pi_{i q}}\right)$ for the multinomial model. For both models, the model parameters are estimated using maximum likelihood. The use of non-canonical link functions requires a constraint in the linear predictors $\left(\eta_{i} \geq 0\right.$ and $\left.\eta_{i j} \geq 0\right)$ to ensure that the disability probabilities $\left(\pi_{i}\right.$ and $\left.\pi_{i j}\right)$ are valid, i.e., the probabilities lie between 0 and 1 . In addhaz, this constraint is implemented in the optimization procedure, with an adaptive barrier algorithm (Lange, 2010), by calling constrOptim in R.

\section{Parallel computing for the bootstrap CI}

Besides the option to estimate the confidence intervals for the parameter estimates based on the standard errors obtained by the inverse of the information matrix for the binomial and multinomial 
models (Wald CI), addhaz also offers the user the option to obtain the bootstrap CI based on empirical percentiles of the replicates (Efron and Tibshirani, 1994).

To reduce computation time, parallel computing can be used to obtain the bootstrap CI. By default $\mathrm{R}$ does not use all the cores available on a computer. The basic idea of parallel computing is to split the work to more than one core of the computer, to execute it in parallel, and then to collect the results. Several R packages can be used to implement parallel computation. In addhaz it is implemented by calling the functions boot and boot.ci in the boot package (Canty and Ripley, 2016; Davison and Hinkley, 1997).

\section{Likelihood ratio test}

The package also includes a function to perform the likelihood ratio test to compare two binomial or multinomial nested models that can be used for model selection.

The likelihood ratio test is defined as $-2^{*} \log ($ likelihood model $1 /$ likelihood model 2$)$. The resulting test statistic is assumed to follow a $\chi^{2}$ distribution, with degrees of freedom ( $\mathrm{df}$ ) equal to the difference of the $\mathrm{df}$ between the models. If the test is statistically significant, the model with more variables fits the data significantly better than the model with less variables.

\section{Alternative software}

The original software for the attribution method (Nusselder and Looman, 2004, 2010) was developed in R, but it is not available as an R package, since it focuses on non-R users: an Excel file is used to input the model arguments and this file is called in the $\mathrm{R}$ code. This software is restricted to binary outcomes and it is freely available upon request to the authors. Different from addhaz, a penalty term is added to the binomial likelihood function when $\pi_{i} \leq 0$, to ensure that valid probabilities are obtained. The original software also allows the user to obtain the bootstrap CI for the model parameters and contributions. Additionally, it offers the options: (i) reduced rank regression (RRR) (Yee, 2014) to reduce the number of parameters when interactions between diseases and age groups are of interest; and (ii) model selection, using the likelihood ratio test.

Besides the original software, the parameter estimates of the binomial additive hazard model can be obtained using the R packages stats with glm and logbin with the function logbin (Donoghoe, 2016). In both packages, the log-binomial model, i.e., $\pi_{i}=\exp \left(\eta_{i}\right)$, used to estimate relative risks (Marschner and Gillett, 2012), can be fitted to a transformed version of the response variable $y^{*}=1-y$, with the $\log$ link function. The estimated model parameters should be multiplied by -1 , since $1-\pi_{i}=\exp \left(-\eta_{i}\right)$. However, care should be taken with glm: by specifying the option family = binomial $(\operatorname{link}=\log )$ to fit the log-binomial model, convergence failure may occur with the iterative weighted least squares (IWLS) algorithm when the maximum likelihood estimates (MLE) lie on the boundary of the parameter space. In glm, the IWLS is modified so that if constraints are violated, step-halving is used iteratively until they are respected. Although this should not result in invalid estimates, it may cause difficulty in convergence. An advantage of logbin is that it includes constrained optimization as an option to optimize the binomial log-likelihood function (method = "ab"). This is done by calling constroptim in $\mathrm{R}$ to constrain the parameter space.

Since addhaz was developed with focus on the attribution method, apart from estimating the model parameters for the binomial additive hazard model, it also gives the user the option to obtain the contribution of diseases to the disability prevalence and to obtain bootstrap CI for the parameter estimates and the contributions, using parallel computing to reduce computation time.

To our knowledge, there is no other package available to fit the multinomial additive hazard model. Although it is possible to fit the log-multinomial model (Blizzard and Hosmer, 2007), i.e., $\pi_{i j}=$ $\exp \left(\eta_{i j}\right)$, with the function vglm in VGAM (Yee, 2016), different from the binomial case, no simple transformation of the outcome can be applied to obtain the parameter estimates of the multinomial additive hazard model using the log-multinomial model.

\section{Using the package addhaz}

In this section, the use of the functions BinAddHaz, MultAddHaz, and LRTest in addhaz are illustrated using a subset of the 2013 BNHS available in the package. A selected output of the results is shown. 


\section{Data description}

The Brazilian National Health Survey (BNHS) ("Pesquisa Nacional de Saúde") was a nationally representative survey of the Brazilian adult population ( $\geq 18$ years) with approximately 60,000 individuals, conducted in 2013. A multistage sampling design with simple random sampling (census tracts) and clustering (households and adults) was used. The response rate was $77 \%$. Survey weights were included to take into account the complex design of the sample. Detailed information about the BNHS can be found in previous publications (Szwarcwald et al., 2014; Yokota et al., 2016).

In addhaz, a subset of the BNHS data is available, with women aged $\geq 60$ years $(n=6,294)$ and the following variables: disability as binary and multinomial outcomes, survey weight, age, diabetes, arthritis, and stroke (Table 1).

\begin{tabular}{|c|c|c|c|}
\hline Variable name & Definition & Type & Categories \\
\hline wgt & survey weight & continuous & - \\
\hline age & age group & binary & $\begin{array}{l}0: 60-79 \text { years } \\
1: \geq 80 \text { years }\end{array}$ \\
\hline diab & diabetes & binary & $\begin{array}{l}0: \text { no } \\
1: \text { yes }\end{array}$ \\
\hline arth & arthritis & binary & $\begin{array}{l}0: \text { no } \\
1: \text { yes }\end{array}$ \\
\hline stro & stroke & binary & $\begin{array}{l}0: \text { no } \\
1: \text { yes }\end{array}$ \\
\hline dis.bin & binary disability outcome & binary & $\begin{array}{l}0: \text { no disability } \\
\text { 1: disabled } \\
0: \text { no disability }\end{array}$ \\
\hline dis.mult & multinomial disability outcome & categorical & $\begin{array}{l}\text { 1: mild disability } \\
\text { 2: severe disability }\end{array}$ \\
\hline
\end{tabular}

Table 1: Description of the variables included in the analysis. Brazilian National Health Survey, 2013.

The binomial and multinomial disability outcomes were defined based on five instrumental activities of daily living (IADL): shopping, handling finances, taking own medications, going to the doctor, and using transportation. Participants were asked about the degree of difficulty in performing IADL tasks, with possible answers: "1. Unable", “2. A lot of difficulty", “3. Some difficulty", or "4. No difficulty". The definition of the binary and multinomial outcomes is shown in Table 2. The reference category "No disability" represents answer " 4 " to all IADL questions.

\begin{tabular}{llc}
\hline Outcome & Outcome category & Answer to at least one IADL question \\
\hline Binary & Disabled & 1,2 or 3 \\
\hline \multirow{2}{*}{ Multinomial } & Mild disability & 3 \\
& Severe disability & 1 or 2 \\
\hline
\end{tabular}

Table 2: Definition of the binary and multinomial disability outcomes. Brazilian National Health Survey, 2013. "IADL" refers to instrumental activities of daily living.

A summary of the characteristics of the study population is shown in Table 3. A higher proportion of older women ( $\geq 80$ years), diabetes, arthritis, stroke, and the disease pairs was observed in women with mild and severe disability compared to women without disability.

\section{Examples with binary outcomes}

The function BinAddHaz fits the binomial additive hazard model and estimates the contribution of diseases to the disability burden for binary outcomes in addhaz. To illustrate the use of BinAddHaz, five models were fitted with the binary disability outcome: model 1 - with three diseases (no background and diseases by age); model 2 - with only background by age, with bootstrap CI; model 3 - with only diseases by age; model 4 - with background and diseases by age, with bootstrap CI; model 5 - with two-way interaction between diseases. To illustrate how the LRTest function can be used for model selection, models 2 and 4 are compared. 


\begin{tabular}{|c|c|c|c|c|c|c|c|c|}
\hline \multirow{2}{*}{ Characteristic } & \multicolumn{2}{|c|}{ Total } & \multicolumn{2}{|c|}{ No disability } & \multicolumn{2}{|c|}{ Mild disability } & \multicolumn{2}{|c|}{ Severe disability } \\
\hline & $\mathrm{N}$ & $\%$ & $\mathrm{~N}$ & $\%$ & $\mathrm{~N}$ & $\%$ & $\mathrm{~N}$ & $\%$ \\
\hline \multicolumn{9}{|l|}{ Age (years) } \\
\hline $60-79$ & 5379 & 85.5 & 3946 & 93.5 & 642 & 78.3 & 791 & 63.0 \\
\hline$\geq 80$ & 915 & 14.5 & 273 & 6.5 & 178 & 21.7 & 464 & 37.0 \\
\hline \multicolumn{9}{|l|}{ Diseases } \\
\hline Diabetes & 1243 & 19.7 & 697 & 16.5 & 190 & 23.2 & 356 & 28.4 \\
\hline Arthritis & 1428 & 22.7 & 819 & 19.4 & 211 & 25.7 & 398 & 31.7 \\
\hline Stroke & 286 & 4.5 & 100 & 2.4 & 41 & 5.0 & 145 & 11.6 \\
\hline Diabetes and arthritis & 298 & 4.7 & 135 & 3.2 & 53 & 6.5 & 110 & 8.8 \\
\hline Diabetes and stroke & 91 & 1.4 & 31 & 0.7 & 13 & 1.6 & 47 & 3.7 \\
\hline Arthritis and stroke & 79 & 1.3 & 28 & 0.7 & 7 & 0.9 & 44 & 3.5 \\
\hline
\end{tabular}

Table 3: Characteristics of the study population. Brazilian National Health Survey, 2013. The percentages refer to unweighted proportions, i.e., without taking into account the survey weights.

\section{Model 1 - Binomial model with three diseases}

Before fitting model 1, addhaz and the data can be loaded and the names of the variables can be checked using:

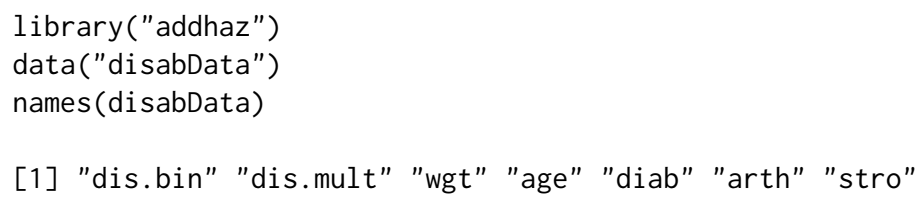

The first binomial model can be fitted with:

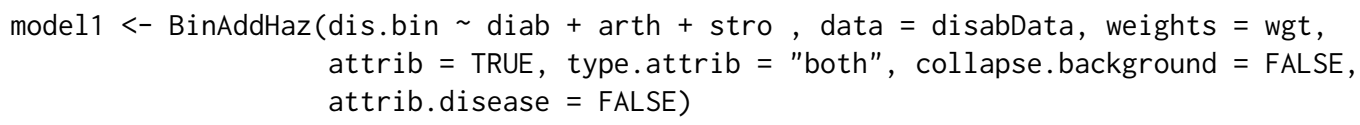

Since no attribution variable such as age was included in the model, the arguments collapse. background and attrib.disease were set to FALSE. The results of the model were stored as an object called model1, which can be checked with the summary function:

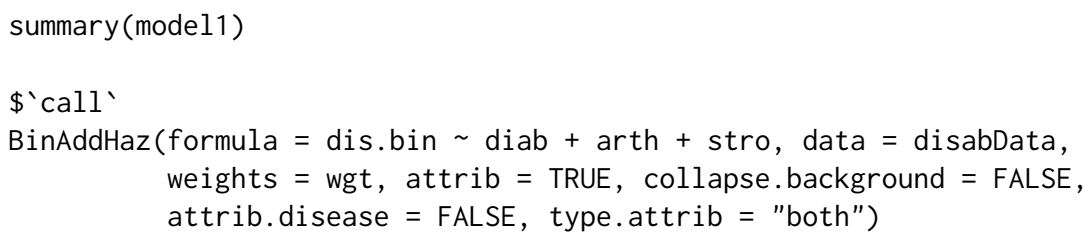

The first element of the output call is the formula used to fit the model. The bootstrap, indicates if the bootstrap CI was requested. Since it was not requested, its value is FALSE.

Next, the coefficients are printed, with their estimates, standard errors (calculated based on the inverse of the observed information matrix), the $t$ value (value of the $t$ statistic), and the $p$ value. The intercept represents the background. According to this output, all diseases were significant in the model. To identify the most disabling diseases, i.e., the diseases with highest cumulative rate of disability, the coefficients can be sorted in decreasing order using: 
sort (model1\$coefficients, decreasing = TRUE)

$\begin{array}{cccc}\text { stro } & \text { (Intercept) } & \text { diab } & \text { arth } \\ .5927519 & 0.2970833 & 0.1331831 & 0.1306445\end{array}$

Stroke was the most disabling disease, while arthritis was the least disabling disease. The 95\% Wald CI can be obtained by:

model1 \$ci

$$
\text { CI2.5 CI97.5 }
$$

(Intercept) $0.27860754 \quad 0.3155590$

$\begin{array}{lll}\text { diab } \quad 0.08649261 & 0.1798735\end{array}$

arth $\quad 0.087125320 .1741637$

stro $\quad 0.444384550 .7411193$

Both the relative and absolute contributions were requested (attrib $=$ TRUE, type. attrib $=$ "both") and can be assessed with:

model1\$contribution

$\${ }^{\prime a t t . r e l}$

att.rel

(Intercept) 0.80405374

diab $\quad 0.06938567$

arth $\quad 0.07451155$

stro $\quad 0.05204903$

\$att.abs

att.abs

disab $\quad 0.30853338$

(Intercept) 0.24807742

diab $\quad 0.02140780$

arth $\quad 0.02298930$

stro $\quad 0.01605886$

In the above output, the relative contribution (att.rel: the contributions sum to 1 ) is shown at the top and the absolute contribution (att.abs: the contributions sum to the disability prevalence) is presented at the bottom. No confidence intervals are provided for the contributions, as they can only be calculated via bootstrapping and this option was not requested.

In the output for the absolute contribution, the disability prevalence (disab) was $30.9 \%$. The absolute contribution can be sorted in decreasing order using:

model1\$contribution\$att.abs[order(model1\$contribution\$att.abs $[, 1]$, decreasing $=\operatorname{TRUE})$,

disab (Intercept) arth diab stro

$\begin{array}{lllll}0.30853338 & 0.24807742 & 0.02298930 & 0.02140780 & 0.01605886\end{array}$

The background (Intercept) was the main contributor to the disability burden in this population. In this case, it can represent other causes not included in the model such as dementia or back pain, which are important causes of disability in the older population, but were not included in the analysis. Among the three diseases, arthritis was the main contributor to the disabilitiy burden in older Brazilian women.

It is interesting to note that, although stroke was the most disabling disease, it showed the lowest contribution to the total disability prevalence. This low contribution can be a consequence of the low occurrence of stroke in this population - $4.5 \%$ (Table 3 ) - as the contribution of chronic conditions to the disability prevalence depends on both, the disease occurrence and the disabling impact (Nusselder and Looman, 2010).

\section{Model 2 - Binomial model with only background by age, with bootstrap CI}

In model 2, the background is modelled by age group, but the diseases are not. The model can be fitted by: 


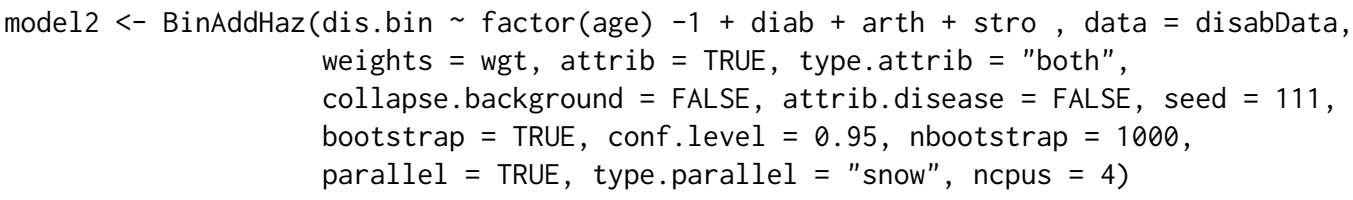

Since the background is modelled by age group, factor(age) is included in the model. The -1 is included in the model. formula to obtain the coefficients for both age groups, including the reference category. Since the background is modelled by age, it should not be collapsed by age (collapse. background $=$ FALSE). As no interaction between diseases and age were included in the model, the argument attrib. disease is FALSE. The option seed $=111$ allows the user to specify a random number to make the results of the bootstrapping reproducible. In the example above, the random number used was 111 . The bootstrap CI for the regression coefficients and the contributions was requested (bootstrap $=$ TRUE), with confidence level $=0.95$ (conf. level $=0.95)$. The bootstrap CI was based on 1000 replicates (nbootstrap $=1000$ ) and it was obatined with parallel computing (parallel $=$ TRUE) on Windows (type. parallel $=$ "snow") with 4 CPUS (ncpus = 4).

The summary of the model can be assessed with:

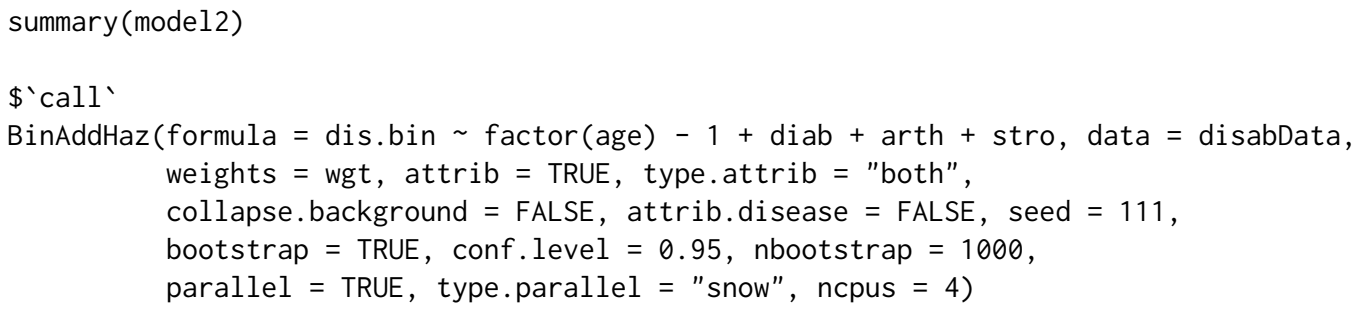

Since the bootstrap CI was requested, bootstrap is TRUE. The coefficients show that age and all diseases were significant (the null value, i.e. 0 , is not included in the bootstrap CI). The factor (age) 0 and factor (age) 1 represents the background cumulative rates for age groups 0 and 1, respectively. The contributions can be checked with:

model2\$contribution

\$att.rel

$\begin{array}{lrrr} & \text { Contribution } & \text { CILow } & \text { CIHigh } \\ \text { factor(age) } 0 & 0.53992912 & 0.51937657 & 0.56054196 \\ \text { factor(age)1 } & 0.29842186 & 0.27575265 & 0.32163433 \\ \text { diab } & 0.06702577 & 0.06162503 & 0.07256112 \\ \text { arth } & 0.04869951 & 0.04513817 & 0.05269298 \\ \text { stro } & 0.04592374 & 0.03666781 & 0.05519789\end{array}$

\$att.abs

Contribution CILow CIHigh

disab $\quad 0.309025460 .302276410 .31623119$

factor(age) $0 \quad 0.16685185 \quad 0.16405831 \quad 0.16947954$

factor(age) $1 \quad 0.09221995 \quad 0.08372162 \quad 0.10131428$

diab $\quad 0.02071267 \quad 0.019069350 .02254047$ 
arth $\quad 0.015049390 .013967440 .01628476$

stro $\quad 0.014191610 .01127267 \quad 0.01727091$

The contributions and the $95 \%$ bootstrap CI are shown. The background is the main contributor to the disability prevalence. Note that by allowing the background to vary by age does not change the disability prevalence $(30.9 \%)$, as compared to model 1 .

\section{Model 3 - Binomial model with only diseases by age}

In Model 3, we allow only the diseases to vary by age group by including interactions between age and diseases in the model. Before fitting model 3, a matrix with the diseases to be included in the model can be defined by:

disease <- as.matrix(disabData[, c("diab", "arth", "stro")])

The first six elements of the matrix created can be checked using:

$\begin{array}{lrrr}\text { head(disease) } & \\ & \text { diab } & \text { arth } & \text { stro } \\ 36 & 1 & 0 & 0 \\ 98 & 0 & 0 & 0 \\ 113 & 0 & 1 & 1 \\ 347 & 1 & 0 & 0 \\ 352 & 1 & 0 & 0 \\ 436 & 0 & 0 & 0\end{array}$

The binomial additive hazard model can be fitted with the function:

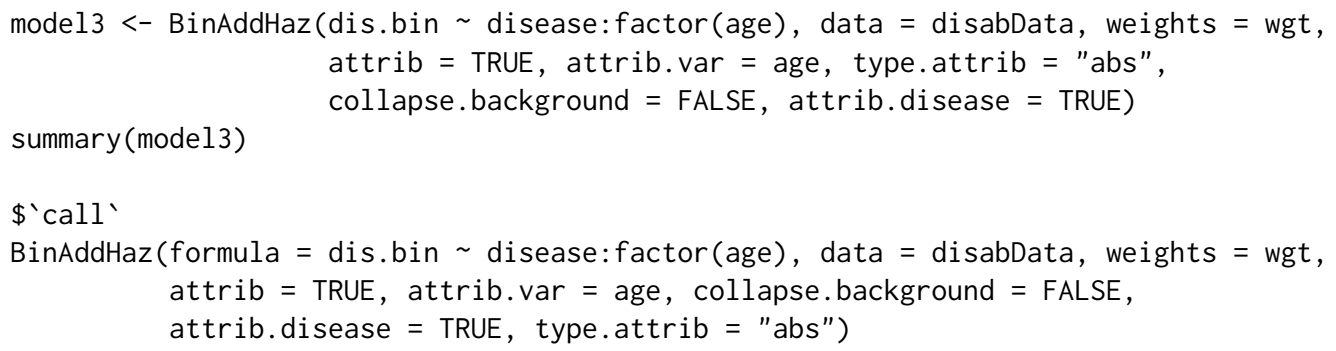

\$bootstrap

[1] FALSE

\$coefficients

$\begin{array}{lrrrr} & \text { Estimate } & \text { StdErr } & \text { t.value } & \text { p.value } \\ \text { (Intercept) } & 0.29425017 & 0.009339432 & 31.5062168 & 1.991333 \mathrm{e}-202 \\ \text { diseasediab: factor (age) } 0 & 0.07487954 & 0.022708458 & 3.2974294 & 9.811601 \mathrm{e}-04 \\ \text { diseasearth: factor (age) } 0 & 0.01218173 & 0.020156904 & 0.6043454 & 5.456358 \mathrm{e}-01 \\ \text { diseasestro: factor(age) } 0 & 0.44896271 & 0.072553106 & 6.1880563 & 6.474653 \mathrm{e}-10 \\ \text { diseasediab:factor(age)1 } & 0.83733434 & 0.128901534 & 6.4959223 & 8.884711 \mathrm{e}-11 \\ \text { diseasearth:factor(age)1 } 1 & 1.32865873 & 0.143790133 & 9.2402636 & 3.299325 \mathrm{e}-20 \\ \text { diseasestro:factor(age)1 } & 1.60530144 & 0.373531351 & 4.2976351 & 1.752351 \mathrm{e}-05\end{array}$

attr (, "class")

[1] "summary. binaddhazmod"

The (Intercept) represents the background, which was not modelled by age. The diseases with factor(age) 0 and factor(age) 1 represent the regression coefficients for age 0 (60-79 years) and age 1 ( $\geq 80$ years). The output above shows that arthritis was not significant for the reference age category (60-79years) (diseasearth: factor (age)0). Only the absolute contribution was requested (type.attrib = "abs") and it can be assessed with:

model3\$contribution

att.abs

disab. 0

0.277835632

backgrnd. 0

0.251005540 
diseasediab: factor (age) $0.0 \quad 0.012575547$

diseasearth: factor (age) $0.0 \quad 0.002220039$

diseasestro: factor (age) $0.0 \quad 0.012034506$

disab. 1

0.493678649

backgrnd.1 0.206353130

diseasediab: factor(age) $1.1 \quad 0.089832332$

diseasearth: factor(age) 1.10 .158378063

diseasestro: factor(age) $1.1 \quad 0.039115125$

The attribution is presented by level of the attribution variable (attrib. var), which in this example is age. The first five rows show the results for attribution variable level 0 , which in this case represents age group 60-79 years and the last five rows represent the results for attribution variable level 1 ( $\geq 80$ years). The results indicate that the disability prevalence in the older women (49.4\%) was much larger than in the younger age group $(27.8 \%)$. While the three diseases included in the model showed a low contribution to the disability prevalence in women aged $60-79$ years $(<1.5 \%)$, arthritis was by far the most important disease contributing to the disability prevalence in the oldest women $(15.9 \%)$.

\section{Model 4 - Binomial model with background and diseases by age, with bootstrap CI}

The same matrix of diseases defined for model 3 will be used in model 4 . This model can be fitted with the function:

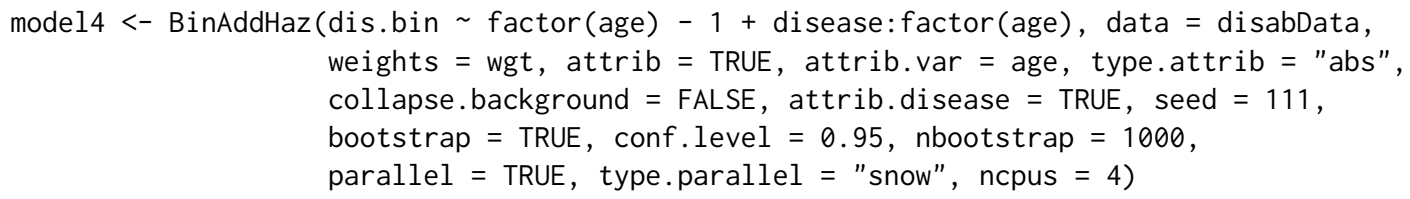

The -1 in the model. formula is used to obtain a different parameterization than the default: here we obtain the parameter estimates for all the age groups, including the reference category. Since we want to estimate the contributions for background by age, the argument collapse. background is set to FALSE. If this argument would be set to TRUE, only one background, common to all age groups, would be estimated. Since the contributions of diseases should be estimated by age group, the argument attrib.disease was set to TRUE. The parallel option for the bootstrap CI was used (parallel = TRUE) on a Windows computer (type. parallel $=$ "snow") with 4 cores $($ ncpus $=4)$. The option seed $=111$ allows the user to specify a random number (in this case 111) to make the results of the bootstrapping reproducible. The summary of the model can be checked with:

summary (model4)

$\$ c a l l$

BinAddHaz (formula = dis. bin $\sim$ factor(age) -1 + disease: factor (age), data = disabData, weights $=$ wgt, attrib = TRUE, attrib.var = age, collapse. background = FALSE, attrib.disease $=$ TRUE, type. attrib $=$ "abs", seed $=111$, bootstrap $=$ TRUE, conf.level $=0.95$, nbootstrap $=1000$, parallel $=$ TRUE, type. parallel = "snow", ncpus $=4$ )

\$bootstrap

[1] TRUE

\$coefficients

$\begin{array}{lrrr} & \text { Estimate } & \text { CILow } & \text { CIHigh } \\ \text { factor(age) } 0 & 0.22661055 & 0.201218693 & 0.2568179 \\ \text { factor(age)1 } & 0.94910725 & 0.784733478 & 1.1292937 \\ \text { factor(age)0: diseasediab } & 0.12749849 & 0.056516120 & 0.2036685 \\ \text { factor(age) 1: diseasediab } & 0.24124648 & -0.181208625 & 0.7471999 \\ \text { factor(age) } 0 \text { : diseasearth } & 0.06884402 & 0.009035558 & 0.1345238 \\ \text { factor(age)1:diseasearth } & 0.75879140 & 0.349882903 & 1.2234047 \\ \text { factor(age) } 0: \text { diseasestro } & 0.48788899 & 0.255772550 & 0.8331633 \\ \text { factor(age)1:diseasestro } & 1.13333515 & 0.426477637 & 2.2240599\end{array}$

$\$$ conf. level

[1] 0.95 
$\operatorname{attr}($, "class")

[1] "summary.binaddhazmod"

The output with the results of the model is shown for factor(age) 0 , which represents the age group of 60-79 years, and factor (age) 1 , representing the age group of $\geq 80$ years. Diabetes was not significant for age group 1 , as the bootstrap CI includes the null value, i.e. 0 . To identify the most disabling diseases, two objects (coef. age 0 and coef. age 1 ) with the coefficients for each age group can be created and sorted in decreasing order using:

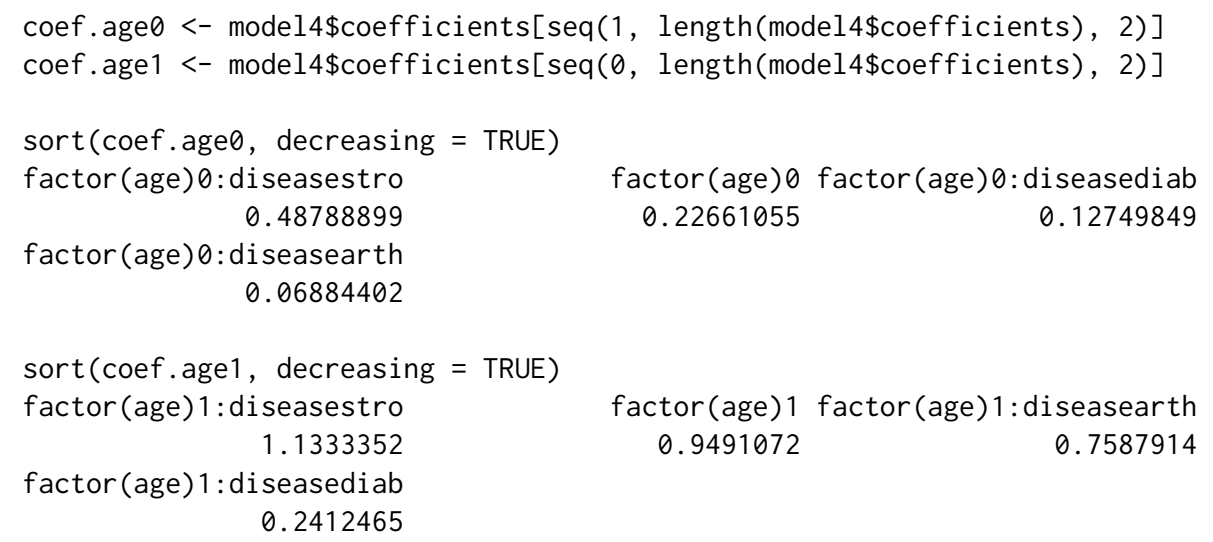

Stroke was the most disabling disease in both age groups. Arthritis and diabetes showed the lowest disabling impact in women aged 60-79 years and $\geq 80$ years, respectively.

Since only the absolute contribution was requested (type.attrib = "abs"), the results for the absolute contribution can be assessed with:

model4 $\$$ contribution

$\begin{array}{lrrr} & \text { att.abs } & \text { CILow } & \text { CIHigh } \\ \text { disab. } 0 & 0.24442949 & 0.24102940 & 0.24813627 \\ \text { backgrnd. } 0 & 0.19743946 & 0.19694283 & 0.19790210 \\ \text { factor(age) } 0 \text { : diseasediab. } 0 & 0.02141352 & 0.01956557 & 0.02342391 \\ \text { factor(age) } 0 \text { :diseasearth. } 0 & 0.01253887 & 0.01153068 & 0.01363192 \\ \text { factor(age) } 0 \text { :diseasestro. } 0 & 0.01303764 & 0.01003208 & 0.01636211 \\ \text { disab.1 } & 0.69484947 & 0.68537515 & 0.70574268 \\ \text { backgrnd.1 } & 0.54868976 & 0.53993448 & 0.55643174 \\ \text { factor(age) 1:diseasediab.1 } & 0.02637877 & 0.02080951 & 0.03249654 \\ \text { factor(age)1:diseasearth.1 } & 0.09137348 & 0.07746954 & 0.10759760 \\ \text { factor(age) 1:diseasestro.1 } & 0.02840746 & 0.01932950 & 0.03894183\end{array}$

The CILow and CIHigh refers to the 2.5th and 97.5th percentiles of the 1,000 bootstrap replicates, since the bootstrap CI was requested (bootstrap $=$ TRUE) with conf. level $=0.95$. To identify the main contributors to the disability burden, two objects (one for each age group) can be defined with the absolute contribution and bootstrap CI using:

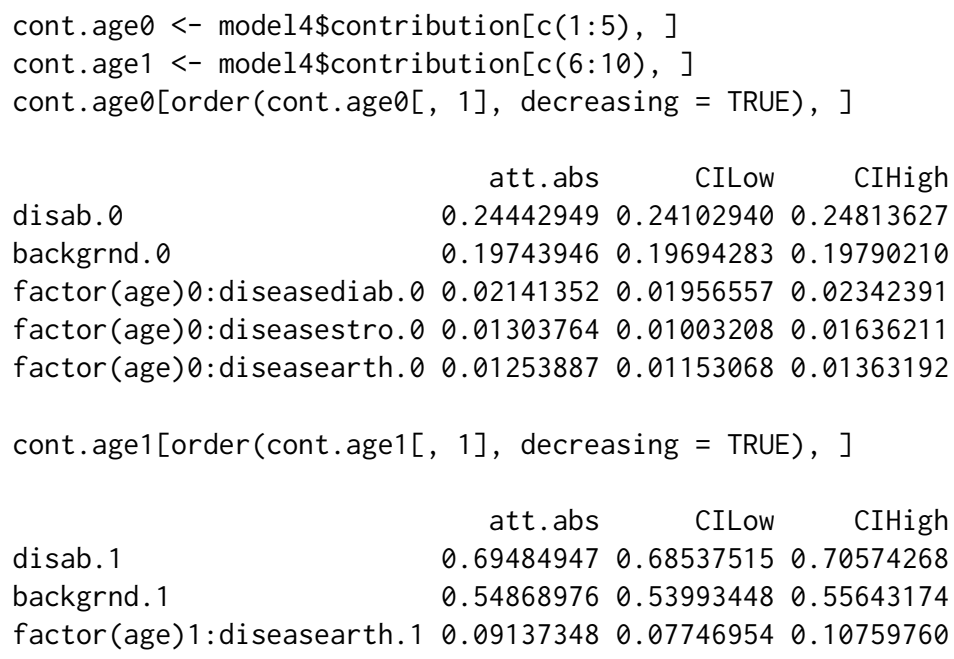


factor(age) 1 : diseasestro. $1 \quad 0.02840746 \quad 0.01932950 \quad 0.03894183$

factor(age) 1 : diseasediab. $1 \quad 0.02637877 \quad 0.02080951 \quad 0.03249654$

According to the results, the disability prevalence in the oldest women $(69.5 \%)$ was 2.8 times larger than in women aged $60-79$ years $(24.4 \%)$. The background was the main contributor to the disability prevalence in both age groups. Among the chronic conditions, diabetes was the main contributor to the disability prevalence in women aged $60-79$ years $(2.1 \%)$ while arthritis contributed most to the disability burden in older women $(9.1 \%)$.

\section{Model 5 - Binomial model with two-way interaction between diseases}

In model 5, the independence assumption (assumption vii) is violated and two-way interactions between diseases are included in the model. In total, 6 parameters and the intercept will be estimated in model 5. The model can be fitted by:

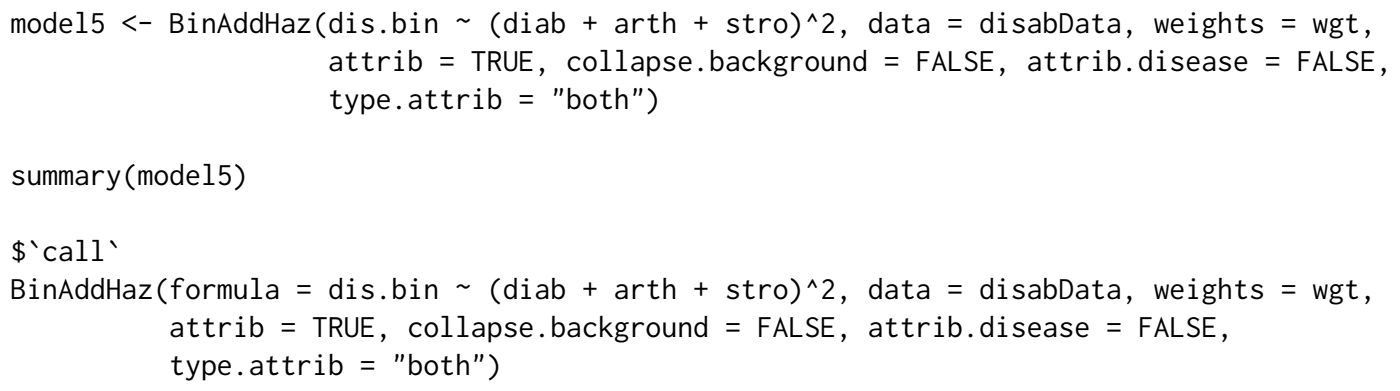

The main effects of all the diseases were significant, but only the interaction between diabetes and arthritis and between diabetes and stroke were significant. The negative disabling impact of the interaction term between diabetes and stroke should be carefully interpreted, as it is based on a small sample size $(n=91)$ (Table 3$)$.

\section{Likelihood ratio test for model selection}

To illustrate the use of the function LRTest to perform the likelihood ratio test (LRT) for model selection, models 2 (model2) and 4 (model4) are compared. The LRT can be performed with:

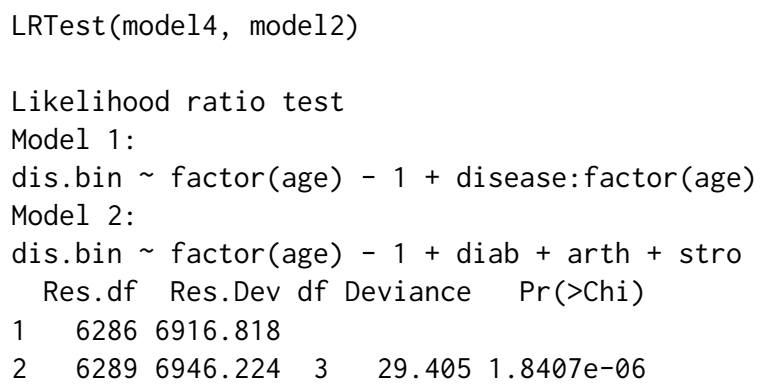

The output shows the models that are being compared: Model 1 is the model with the interactions with diseases and age (previous model 4) and Model 2 is the model without the interactions between 
diseases and age (previous model 2). The degrees of freedom for each model (Res.df), the residual deviance, i.e. the $2 * \log$-likelihood of each model (Res.Dev), the difference in the degrees of freedom between the models $(\mathrm{df})$, the difference between the ${ }^{*} \log$-likelihood of the models, i.e. the value of the likelihood ratio test statistic (Deviance), and the p-value of the test statistic, based on the $\chi^{2}$ distribution $(\operatorname{Pr}(>\mathrm{Chi}))$ are presented. Since the test was statistically significant at 0.05 significance level, model 4, which includes interaction between diseases and age, fits the data better than model 2.

\section{Examples with multinomial outcomes}

To fit the multinomial additive hazard model and to estimate the contribution of chronic conditions to the disability burden for multinomial outcomes, the function MultAddHaz can be used. As an illustration, two models were fitted: model 6 - with only background by age; and model 7 - with background and diseases by age, with bootstrap CI.

\section{Model 6 - Multinomial model with only background by age}

Model 6 can be fitted with the function:

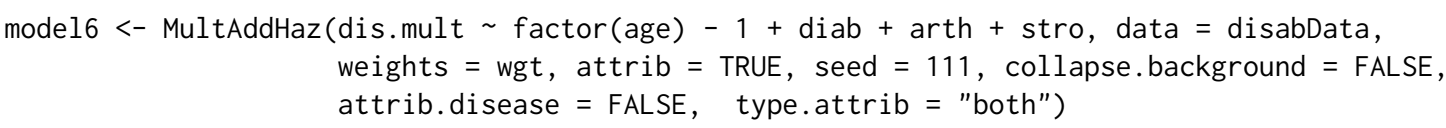

The results of the model can be visualized using:

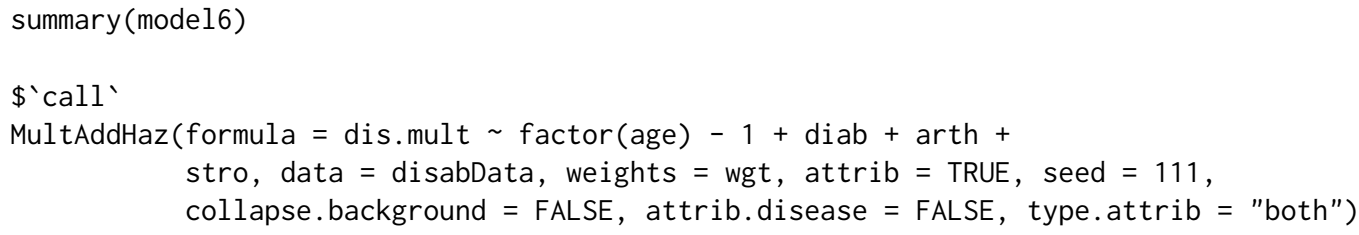

In the above output, the results identified with $y 1$ refer to the outcome (dis.mult) $=1$ (mild disability) and the results with $y 2$ refer to the outcome (dis.mult) $=2$ (severe disability). For mild disability only the background (factor (age)0.y1) and (factor (age)1.y1) was significant while all the diseases and the background were signifcant for women with severe disability. Similar to the binomial model, the most disabling diseases can be identified by:



sort (coef.sev, decreasing $=$ TRUE $)$ 

factor (age)1.y2
stro.y2
diab.y2 factor(age)0.y2
arth.y2
0.81704318
0.49926285
0.12432677
0.10764380
0.06376796

Background and stroke showed the highest disabling impact for mild and severe disability. The relative and absolute contributions can be checked with:

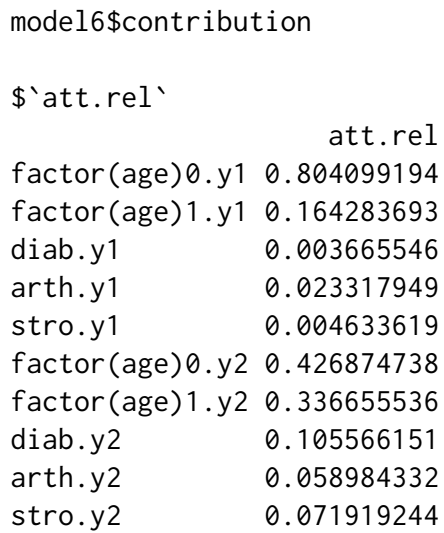

It is interesting to note that the severe disability prevalence (19.0\%) was 1.5 times higher than the mild disability prevalence $(12.7 \%)$. The results for the relative contribution can be sorted in decreasing order by:

rel.cont.mild <- model6\$contribution\$att.rel[1:5, ]

rel.cont.sev <- model6\$contribution\$att.rel[6:10, ]

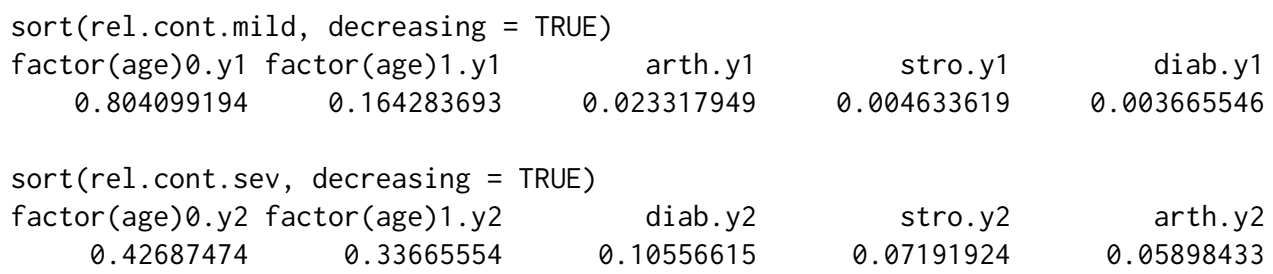
0.164283693
0.023317949
0.004633619
0.003665546

0.804099194

sort (rel. cont.sev, decreasing = TRUE)
factor (age) 0 .y 2 factor (age) $1 . y 2$
diab.y2
stro.y2
arth.y2
0.42687474
0.33665554
0.10556615
0.07191924
0.05898433

The background was the main contributor to the disability burden, representing $96.8 \%(0.80+0.16)$ and $76.4 \%(0.43+0.34)$ of the mild and severe disability prevalence, respectively. Arthritis $(2.3 \%)$ was the main contributor to the mild disability prevalence while diabetes $(10.6 \%)$ contributed most to the severe disability prevalence.

\section{Model 7 - Multinomial model with background and diseases by age, with bootstrap CI}

The matrix with the diseases (disease) defined for model 3 is used to fit model 7:

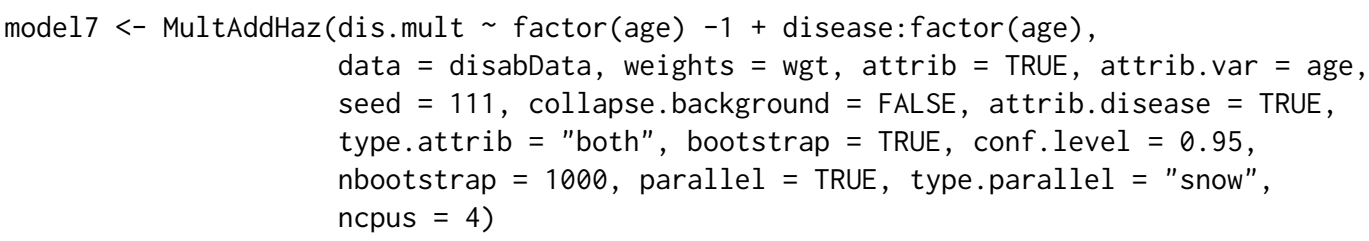


The -1 was added to the model. formula to obtain the parameter estimates for the background for all age groups, including the reference category. Since the background should be estimated by age, collapse. background is set to FALSE. Additionally, attrib.disease is set to TRUE, as interactions between age and diseases were included in the model and the contribution of diseases should be estimated by age. The seed argument in MultAddHaz is used to obtain reproducible results for the starting values used in the constrained optimization, which are randomly generated, and for the bootstrap CI. Besides the summary function, the disabling impacts and the bootstrap CI can also be assessed with:

cbind(model7 $\$$ coefficients, model $7 \$ c i)$

$\begin{array}{lrrr} & \text { Coefficients } & \text { CILow } & \text { CIHigh } \\ \text { factor(age) } 0 . y 1 & 0.117255379 & 0.10064630 & 0.13689281 \\ \text { factor(age)1.y1 } & 0.277818866 & 0.20558349 & 0.37304023 \\ \text { factor(age) } 0: \text { diseasediab.y1 } & 0.005926107 & -0.03440926 & 0.04770883 \\ \text { factor(age)1:diseasediab.y1 } & -0.027900849 & -0.16964726 & 0.15898841 \\ \text { factor(age) } 0: \text { diseasearth.y1 } & 0.012814735 & -0.02467113 & 0.05156243 \\ \text { factor(age)1:diseasearth.y1 } & 0.110733103 & -0.08447433 & 0.30975351 \\ \text { factor(age) } 0: \text { diseasestro.y1 } & 0.032163873 & -0.03657685 & 0.14572706 \\ \text { factor(age)1:diseasestro.y1 } & -0.028504716 & -0.22807053 & 0.22952796 \\ \text { factor(age) } 0 . y 2 & 0.109165655 & 0.09146724 & 0.13167070 \\ \text { factor(age)1.y2 } & 0.672941316 & 0.53792263 & 0.83185332 \\ \text { factor(age) } 0: \text { diseasediab.y2 } & 0.121508443 & 0.06618176 & 0.17864913 \\ \text { factor(age)1:diseasediab.y2 } & 0.282986455 & -0.09742139 & 0.80712949 \\ \text { factor(age) } 0: \text { diseasearth.y2 } & 0.054335292 & 0.01095538 & 0.09957643 \\ \text { factor(age)1:diseasearth.y2 } & 0.635463641 & 0.31671319 & 1.05424237 \\ \text { factor(age) } 0: \text { diseasestro.y2 } & 0.456594023 & 0.24959719 & 0.72863913 \\ \text { factor(age)1:diseasestro.y2 } & 1.233578243 & 0.49988818 & 2.27216717\end{array}$

In the output, factor (age) 0 and factor (age) 1 refers to the age groups $60-79$ years and $\geq 80$ years, respectively. $\mathrm{y} 1$ refers to disability category 1 , which here represents mild disability and y 2 refers to disability category 2 , representing severe disability.

Two coefficients (for diabetes and stroke in women aged $\geq 80$ years with mild disability) were negative. This suggests a "protective" effect of these conditions. However, these results should be carefully interpreted as they were not statistically significant.

To identify the most disabling diseases for mild and severe disability by age group, the following code can be used:

mild.age $0<-$ model7\$coefficients[seq(1, length(model7\$coefficients), 2), ][1:4] sev.age $0<-$ model7\$coefficients[seq(1, length(model7\$coefficients), 2), ][5:8] mild.age $1<-\operatorname{model} 7$ \$coefficients $[$ seq $(\theta$, length(model7\$coefficients), 2), ][1:4] sev.age $1<-$ model7 $\$$ coefficients $[\operatorname{seq}(0$, length(model7\$coefficients), 2), ][5:8]

mild.age $0[\operatorname{order}(\operatorname{mild}$.age 0 , decreasing $=$ TRUE) $]$

factor (age) $0 . y 1$ factor (age) 0 : diseasestro.y 1
0.117255379
0.032163873

factor (age) 0 : diseasearth.y1 factor (age) 0 : diseasediab. y1

$$
0.012814735 \quad 0.005926107
$$

sev.age 0 [order (sev.age 0 , decreasing = TRUE)]

factor(age) 0 : diseasestro.y2 factor(age) 0 : diseasediab.y2
0.45659402
0.12150844

factor (age) 0. y2 factor (age) 0 : diseasearth. y2
0.10916565
0.05433529

mild.age1[order(mild.age1, decreasing = TRUE)]

factor (age) $1 . y 1$ factor (age) 1 : diseasearth. y1
0.27781887
0.11073310

factor (age) 1: diseasediab.y1 factor (age) 1: diseasestro.y1

$$
-0.02790085 \quad-0.02850472
$$

sev.age1[order (sev.age1, decreasing = TRUE)]

factor(age) 1: diseasestro.y2 factor(age)1.y2
1.2335782
0.6729413 
factor(age) 1: diseasearth.y2 factor(age)1: diseasediab.y2

$$
0.6354636 \quad 0.2829865
$$

Stroke was the most disabling disease in women with severe disability in both age groups and in women aged 60-79 years with mild disability while arthritis was the most disabling disease in women aged $\geq 80$ years with mild disability.

The main contributors to the disability burden, based on the absolute contribution can be assessed with:

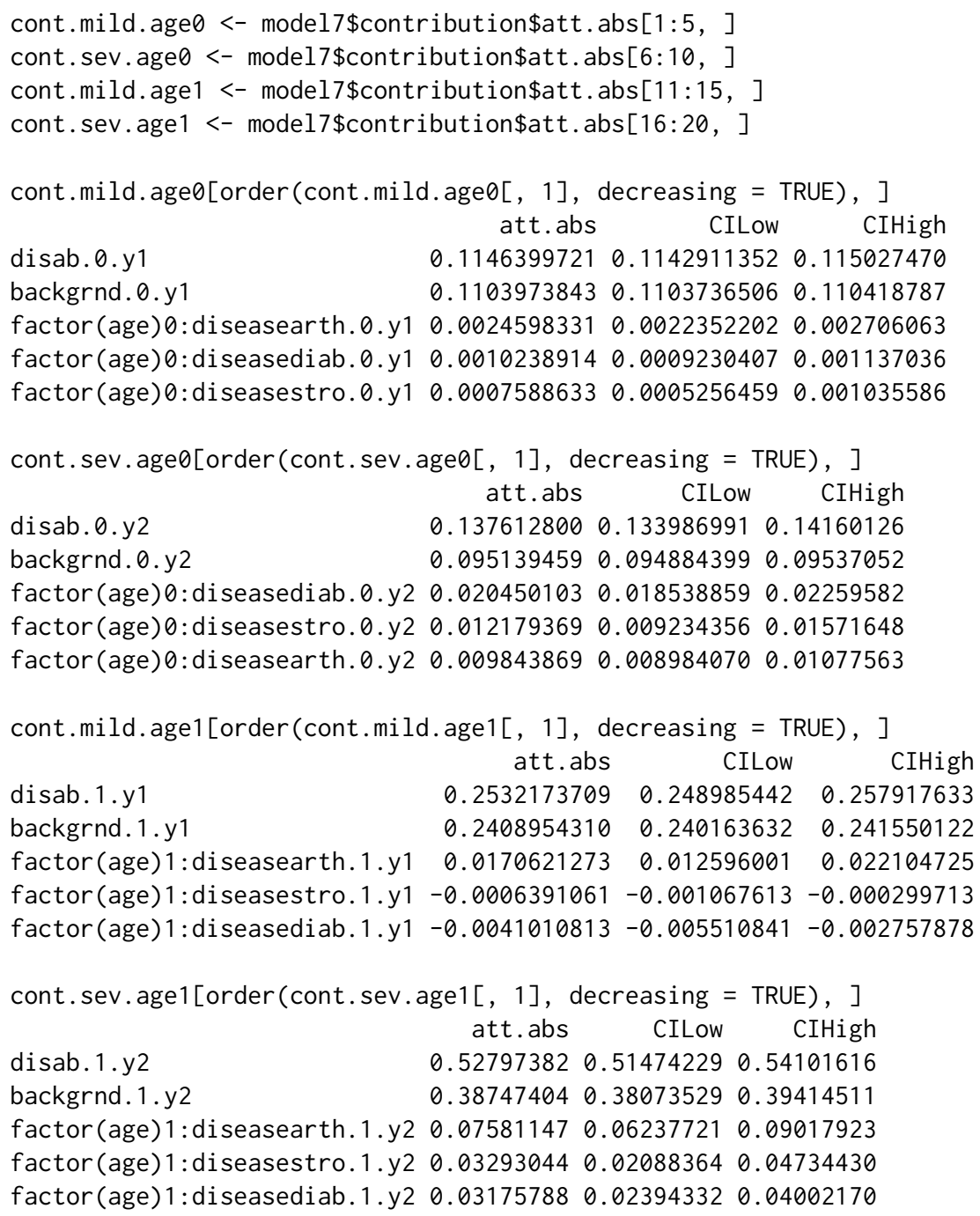

The severe disability prevalence $(60-79$ years $=13.8 \% ; \geq 80$ years $=52.8 \%)$ was larger than the mild disability prevalence $(60-79$ years $=11.5 \%$; $\geq 80$ years $=25.3 \%)$ in both age groups. Arthritis was the main contributor to the mild disability prevalence in both age groups and to the severe disability prevalence in women aged $\geq 80$ years, while diabetes was the main contributor to the severe disability prevalence in women aged 60-79 years.

\section{Discussion}

In this paper we introduced the $\mathrm{R}$ package addhaz developed to fit the binomial and multinomial additive hazard models to estimate the contribution of diseases to the disability prevalence using cross-sectional data.

The R package addhaz was developed based on the R functions developed by Nusselder and Looman (Nusselder and Looman, 2010) for binomial disability outcomes and for non-R users. The main advantages of addhaz compared to the original $R$ functions are: (i) option to use the attribution method for multinomial responses using the function MultAddHaz; and (ii) option to do parallel computing for the calculation of the bootstrap percentile confidence intervals. However, the possibility to use reduced rank regression (Yee, 2014) to estimate the cause-specific disability rates by age group, 
for example, which is available in the original $\mathrm{R}$ functions (Nusselder and Looman, 2010), is not available in addhaz. Nonetheless, in addhaz these interactions can be estimated by including full interaction terms between chronic conditions and age groups.

Although the parameter estimates of the binomial additive hazard model can also be obtained with the R package logbin, the contribution of diseases to the disability prevalence is not provided, since logbin was not developed with focus on the attribution method. Therefore, for analysis aimed at the attribution of disability to diseases, we recommend the use of addhaz. For multinomial outcomes, no other software is available to fit the multinomial additive hazard model and to calculate the contributions, to our knowledge.

One could argue that instead of using the multinomial model, two binomial models could be fitted: (i) no x mild disability; and (ii) no x severe disability. Although this is indeed possible, with a minor loss of precision (larger standard errors) for the parameter estimates when the reference category ("no disability", in our example) is the most frequent category in the population (which is the case for the subset of the BNHS used here, as $67 \%$ were not disabled, $13 \%$ reported mild disability, and $20 \%$ were severely disabled) (Agresti, 2002), the prevalence of the various disability categories do not sum to $100 \%$, as can be observed in a previous study that assessed the difference in the mild and severe disability burden using two binomial models (Yokota et al., 2015a).

Different models with different options were presented using addhaz, showing a wide possibility of application to the users. One example is the investigation of the role of multimorbidity on the disability prevalence, which was assessed by the inclusion of two-way interactions between diseases in the model, as presented in model 2. Even though the examples only included the combination of two diseases, higher order interactions can also be included in the models, with the sample size being the limiting factor. In addition, since the prevalence of chronic conditions tends to increase over age, the model parameterization to include interactions between diseases and age groups was also shown for the binary (models 3 and 4) and multinomial disability outcomes (model 7). Although age group was used as the stratification variable to estimate the disabling impacts of the diseases and background, other variables can be used, such as education attainment and sex.

Furthermore, we illustrated how the likelihood ratio test (LRT) can be performed for model selection using the function LRTest. The LRT can be also performed for model selection with the multinomial additive hazard model.

The attribution method has some limitations that should be considered. The main limitation of the method is the causality assumption. Although a causal relationship between diseases and disability is plausible and has been proposed in several disability models (Verbrugge and Jette, 1994), it cannot be assessed with cross-sectional data. As a consequence, disability is incorrectly attributed to diseases when disability occurred before the diseases. Although the parallel option reduces significantly computation time for calculating bootstrap confidence intervals, fitting the multinomial model to high dimensional data can still be time-consuming. For example, the computational time to fit model 7, in a Windows computer Intel(R) Core (TM) i7-4600 CPU with 2.1GHz and 2.7GHz, 8GB (RAM), using the parallel option with 4 cores, was 23.04 hours.

\section{Summary}

In conclusion, addhaz is a publicly available tool to assess the contribution of chronic conditions to the disability prevalence, using cross-sectional data. The results produced by the tool can be used by policymakers to reduce the disability burden. Future areas of interest to improve the package include the extension of the multinomial model to ordinal responses and alternatives to reduce computation time for high dimensional data.

\section{Bibliography}

A. Agresti. Categorical Data Analysis. John Wiley \& Sons, 2002. ISBN 0-471-36093-7. [p92]

J. R. Beard, A. Officer, I. A. de Carvalho, R. Sadana, A. M. Pot, J.-P. Michel, P. Lloyd-Sherlock, J. E. Epping-Jordan, G. G. Peeters, W. R. Mahanani, et al. The world report on ageing and health: A policy framework for healthy ageing. The Lancet, 387(10033):2145-2154, 2016. URL http://dx. doi . org/10.1016/S0140-6736(15)00516-4. [p75]

L. Blizzard and D. Hosmer. The log multinomial regression model for nominal outcomes with more than two attributes. Biometrical Journal, pages 889-902, 2007. URL https://doi . org/10.1002/bimj . 200610377. [p79] 
A. Canty and B. Ripley. boot: Bootstrap R (S-PLUS) Functions, 2016. URL https: //CRAN. R-project . org/package=boot. R package version 1.3-18. [p79]

H. Chen, H. Wang, E. M. Crimmins, G. Chen, C. Huang, and X. Zheng. The contributions of diseases to disability burden among the elderly population in china. Journal of Aging and Health, pages 261-82, 2013. URL https://doi.org/10.1177/0898264313514442. [p75]

C. Chiang. Competing risks in mortality analysis. Annual Review of Public Health, pages 281-307, 1991. URL https://doi.org/10.1146/annurev.pu.12.050191.001433. [p77]

A. Davison and D. Hinkley. Bootstrap Methods and Their Applications. Cambridge University Press, 1997. ISBN 0-521-57391-2. [p79]

M. W. Donoghoe. logbin: Relative Risk Regression Using the Log-Binomial Model, 2016. URL https: //CRAN.R-project.org/package=logbin. R package version 2.0.1. [p79]

B. Efron and R. J. Tibshirani. An Introduction to the Bootstrap. Chapman \& Hall/CRC Press, 1st edition, 1994. ISBN 0412042312. [p76, 78, 79]

B. Klijs, W. J. Nusselder, C. W. Looman, and J. P. Mackenbach. Contribution of chronic disease to the burden of disability. PLoS One, 6(9):e25325, 2011. URL https://doi .org/10.1371/journal . pone. 0025325. [p75]

K. Lange. Numerical Analysis for Statisticians. Springer-Verlag, 2nd edition, 2010. ISBN 978-1-4419-59447. [p78]

K. Manton and E. Stallard. Recent Trends in Mortality Analysis. Academic Press, 1984. ISBN 0124700209. [p77]

I. Marschner and A. Gillett. Relative risk regression: Reliable and flexible methods for log-binomial models. Biostatistics, pages 179-192, 2012. URL https://doi .org/10.1093/biostatistics/kxr030. [p79]

W. Nusselder and C. Looman. WP7: Decomposition tools - Technical report on attribution tool. Technical report, 2010. URL http://www. eurohex.eu/pdf/Reports\{_\}2010/2010TR7. $2\left\{\_\right\}$TRonattributiontool.pdf. [p75, 76, 77, 79, 82, 91, 92]

W. J. Nusselder and C. W. Looman. Decomposition of differences in health expectancy by cause. Demography, 41(2):315-334, 2004. URL https://doi .org/10.1353/dem.2004.00. [p75, 76, 77, 79]

W. J. Nusselder, C. W. Looman, J. P. Mackenbach, M. Huisman, H. Van Oyen, P. Deboosere, S. Gadeyne, and A. E. Kunst. The contribution of specific diseases to educational disparities in disability-free life expectancy. American Journal of Public Health, 95(11):2035-2041, 2005. URL http://doi . org/10. 2105/AJPH. 2004.054700. [p75]

SAS Institute Inc. The sas system, version 9.2, 2008. URL http://www. sas. com/. [p75]

R. Strobl, M. Müller, R. Emeny, A. Peters, and E. Grill. Distribution and determinants of functioning and disability in aged adults-results from the german kora-age study. BMC Public Health, 13(1):1, 2013. URL http://doi.org/10.1186/1471-2458-13-137. [p75]

C. Szwarcwald, D. Malta, C. Pereira, M. Vieira, W. Conde, P. Souza Júnior, G. Damacena, L. Azevedo, G. Silva, M. Theme Filha, C. Lopes, D. Romero, W. Almeida, and C. Monteiro. Pesquisa nacional de saúde no brasil: Concepção e metodologia de aplicação. Ciência E Saúde Coletiva, 19(2):333-342, 2014. URL http://dx.doi.org/10.1590/1413-81232014192.14072012. [p80]

L. Verbrugge and A. Jette. The disablement process. Social Science \& Medicine, pages 1-14, 1994. URL http://dx.doi.org/10.1016/0277-9536(94)90294-1. [p92]

M. Yang, X. Ding, and B. Dong. The measurement of disability in the elderly: A systematic review of self-reported questionnaires. Journal of the American Medical Directors Association, 15(2):150-e1, 2014. URL https://doi.org/10.1016/j. jamda.2013.10.004. [p75]

T. Yee. Reduced-rank vector generalized linear models with two linear predictors. Computational Statistics E Data Analysis, pages 889-902, 2014. URL https://doi.org/10.1016/j.csda. 2013.01. 012. [p79,91]

T. W. Yee. VGAM: Vector Generalized Linear and Additive Models, 2016. URL https://CRAN. R-project. org/package=VGAM. R package version 1.0-2. [p79] 
R. Yokota, J. Van der Heyden, S. Demarest, J. Tafforeau, W. Nusselder, P. Deboosere, and H. Van Oyen. Contribution of chronic diseases to mild and severe disability burden in Belgium. Archives of Public Health, page 37, 2015a. URL http://doi .org/10.1186/s13690-015-0083-y. [p92]

R. T. C. Yokota, N. Berger, W. J. Nusselder, J.-M. Robine, J. Tafforeau, P. Deboosere, and H. Van Oyen. Contribution of chronic diseases to the disability burden in a population 15 years and older, Belgium, 1997-2008. BMC Public Health, 15(1):1, 2015b. URL https://doi .org/10. 1186/s12889-015-1574-z. [p75]

R. T. C. Yokota, L. de Moura, S. S. C. de Araújo Andrade, N. N. B. de Sá, W. J. Nusselder, and H. Van Oyen. Contribution of chronic conditions to gender disparities in disability in the older population in Brazil, 2013. International Journal of Public Health, 61(9):1003-1012, 2016. URL https: //doi.org/10.1007/s00038-016-0843-7. [p75, 80]

R. T. C. Yokota, H. Van Oyen, C. W. N. Looman, W. J. Nusselder, M. Otava, Y. W. Kifle, and G. Molenberghs. Multinomial additive hazard model to assess the disability burden using cross-sectional data. Biometrical J., 2017. URL https://doi .org/10.1002/bimj.201600157. [p75]

Renata Tiene de Carvalho Yokota

Epidemiology and Public Health, Sciensano

Interface Demography, Vrije Universiteit Brussel

Belgium

Renata.Yokota@sciensano.be

Caspar W. N. Looman

Department of Public Health, Erasmus Medical Center

Netherlands

c. looman@erasmusmc.n1

Wilma Johanna Nusselder

Department of Public Health, Erasmus Medical Center

Netherlands

w.nusselder@erasmusmc.nl

Herman Van Oyen

Epidemiology and Public Health, Sciensano

Department of Public Health, Universiteit Gent

Belgium

Herman.VanOyen@sciensano. be

Geert Molenberghs

Interuniversity Institute for Biostatistics and statistical Bioinformatics (I-BioStat), Universiteit Hasselt and Katholieke Universiteit Leuven

Belgium

geert.molenberghs@uhasselt.be 